\title{
THE MINIMAL NORMAL $\mu$-COMPLETE FILTER ON $P_{\kappa} \lambda$
}

\author{
MASAHIRO SHIOYA
}

(Communicated by Andreas R. Blass)

\begin{abstract}
We introduce the closed (resp. strongly closed) $\mu$-unbounded filter $C F_{\kappa \lambda}^{\mu}$ (resp. $S C F_{\kappa \lambda}^{\mu}$ ) for a regular cardinal $\mu \leq \kappa$, which is a $\mu$-complete analog of the closed (resp. strongly closed) unbounded filter on $P_{\kappa} \lambda$. We give their simple characterizations for the case $\mu=\omega_{1}$. We also study generators of $C F_{\kappa \lambda}^{\nu}$ (resp. $S C F_{\kappa \lambda}^{\nu}$ ) over $C F_{\kappa \lambda}^{\mu}$ (resp. $S C F_{\kappa \lambda}^{\mu}$ ) for the case $\omega<\mu<\nu$.
\end{abstract}

\section{INTRODUCTION}

The closed unbounded filter on $P_{\kappa} \lambda$, denoted by $C F_{\kappa \lambda}$, was introduced by Jech [5] as a natural generalization of that on a regular cardinal $\kappa$. Its fundamental characterization as the minimal normal $\kappa$-complete filter was established by Carr [1]. At the same time, she reformulated a result of Menas [8] which indicates the complexty of $P_{\kappa} \lambda$ relative to $\kappa$, as the difference between $C F_{\kappa \lambda}$ and the strongly closed unbounded filter $S C F_{\kappa \lambda}$ introduced by herself. This simple fact motivates our work in the present paper and [10].

In this note, we introduce their $\mu$-complete analogs for a regular cardinal $\mu \leq \kappa$, i.e., the closed $\mu$-unbounded filter $C F_{\kappa \lambda}^{\mu}$ and the strongly closed $\mu$ unbounded filter $S C F_{\kappa \lambda}^{\mu}$. Our main concern is to stress the difference between $C F_{\kappa \lambda}^{\mu}$ and $S C F_{\kappa \lambda}^{\mu}$ through investigating the forms of their generators.

First we consider the case $\mu=\omega_{1}$. For their own purposes, several people [2, $4,6,9]$ have already considered similar problems for $C F_{\kappa \lambda}$, or more specifically for $C F_{\omega_{1} \lambda}$. Among them, Matet [7] gave the simplest solutions to both cases. By a rather different argument from his, we show that $C F_{\kappa \lambda}^{\omega_{1}}$ is generated by the sets of the form $\left\{x: f^{\prime \prime} x^{2} \subset x\right\}$, where $f: \lambda^{2} \rightarrow \lambda$. This includes both his results as immediate corollaries. We also show that $S C F_{\kappa \lambda}^{\omega_{1}}$ can be characterized by two unary functions on $\lambda$ but not by one unary function, in contrast to $C F_{\kappa \lambda}^{\omega_{1}}$.

The same problem as above is meaningless for the case $\mu>\omega_{1}$. Instead we study generators of $C F_{\kappa \lambda}^{\nu}$ (resp. $S C F_{\kappa \lambda}^{\nu}$ ) over $C F_{\kappa \lambda}^{\mu}$ (resp. $S C F_{\kappa \lambda}^{\mu}$ ) for

Received by the editors April 7, 1992 and, in revised form, August 9, 1993.

1991 Mathematics Subject Classification. Primary 03E05.

Key words and phrases. $P_{\kappa} \lambda, C F_{\kappa \lambda}^{\mu}, S C F_{\kappa \lambda}^{\mu}$.

The results in the present paper are taken from author's Ph.D. thesis written under the guidance of Professor K. Eda, to whom the author is very grateful.

This research was partially supported by Grant-in-Aid for Scientific Research (No. 04302009), Ministry of Education, Science and Culture. 


\section{TABLE 1}

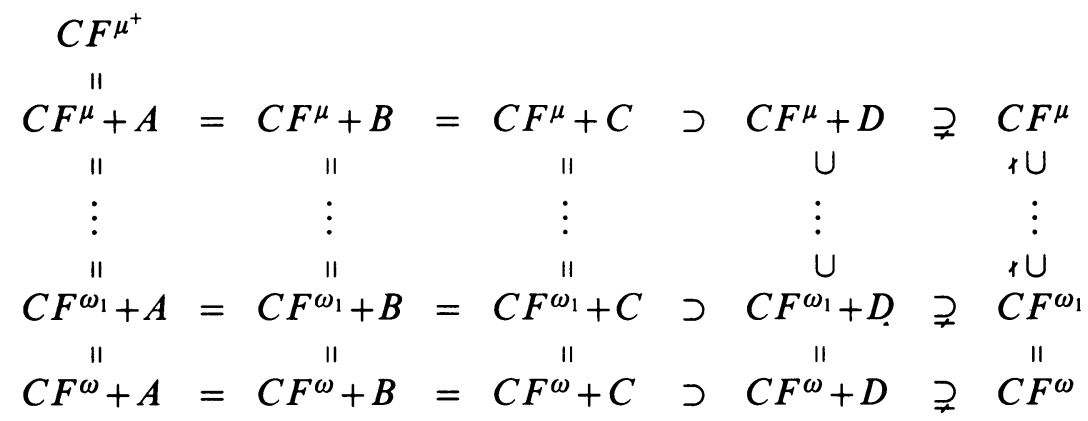

\section{$S C F^{\mu^{+}}$}

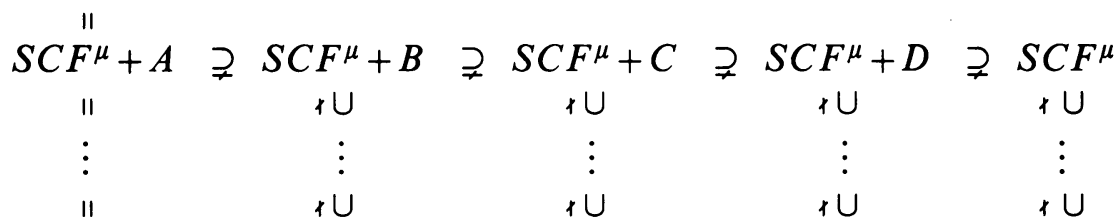

$S C F^{\omega_{1}}+A \supsetneq S C F^{\omega_{1}}+B \supsetneq S C F^{\omega_{1}}+C \supsetneq S C F^{\omega_{1}}+D \supsetneq S C F^{\omega_{1}}$

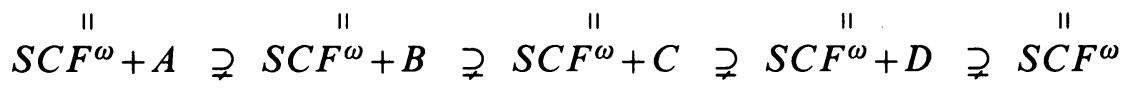

$\omega<\mu<\nu$. For example, it has been known to people including authors of [2, 4] that the set $\{x: x \cap \kappa \in \kappa\}$ generates $C F_{\kappa \lambda}$ over $C F_{\kappa \lambda}^{\omega_{1}}$. We investigate whether this statement would hold for other simply definable subsets of $P_{\kappa} \lambda$.

Table 1 summarizes our result. Subscripts $\kappa$ and $\lambda$ are dropped for notational simplicity. We also abbreviate by $A, B, C$, and $D$ the sets $\left\{x: \sigma_{\mu}^{\prime \prime} x \subset\right.$ $P x\},\left\{x: x \cap \mu^{+} \in \mu^{+}\right\},\{x: \mu \subset x\}$, and $\{x:|x| \geq \mu\}$ respectively, where $\omega_{1}<\mu<\kappa$.

\section{Preliminaries}

Fix a regular cardinal $\kappa>\omega$ and a cardinal $\lambda>\kappa$. We begin by recalling some basic notions on $P_{\kappa} \lambda=\{x \subset \lambda:|x|<\kappa\} . F \subsetneq P P_{\kappa} \lambda$ is called a filter if it is closed under intersections and supersets and is fine (i.e., $\{x: \alpha \in x\} \in F$ for any $\alpha<\lambda)$. A subset of $P_{\kappa} \lambda$ is said to be closed (resp. strongly closed) if it is closed under unions of chains (resp. subsets) of cardinality $<\kappa$, and is said to be unbounded if it is cofinal in the partially ordered set $\left(P_{\kappa} \lambda, \subset\right)$. A filter $F$ is said to be $\mu$-complete if it is closed under intersections of subsets of cardinality $<\mu$ and is said to be normal if it is closed under diagonal intersections (i.e., $\Delta_{\alpha<\lambda} X_{\alpha}=\left\{x: \forall \alpha \in x \quad x \in X_{\alpha}\right\} \in F$ for any $\left.\left\{X_{\alpha}: \alpha<\lambda\right\} \subset F\right) . C F_{\kappa \lambda}$ (resp. $S C F_{\kappa \lambda}$ ) is the filter generated by the closed (resp. strongly closed) and unbounded subsets of $P_{\kappa} \lambda$.

We use $\mu$ and $\nu$ (resp. $\xi$ and $\zeta$ ) to denote an infinite regular cardinal $\leq \kappa$ (resp. an uncountable cardinal $<\kappa$ ). We denote by $F+X$ the filter generated by $F \cup\{X\}$, where $X \subset P_{\kappa} \lambda$ is positive and copositive with respect to a filter $F$ (i.e., $\left.P_{\kappa} \lambda-X, X \notin F\right) . \alpha+\beta$ (resp. $\alpha \cdot \beta$ ) is the ordinal sum 
(resp. product) of $\alpha$ and $\beta$. We fix a bijection $\pi: \lambda^{2} \rightarrow \lambda$. $\sigma: \lambda \rightarrow \lambda$ and $\sigma_{\xi}: \lambda \rightarrow[\lambda]^{\xi}$ denote the successor function and the function defined by $\sigma_{\xi}(\alpha)=\{\alpha+\beta: \beta<\xi\}$ respectively. By $\alpha \rightarrow[\beta]_{P_{\gamma} \delta}^{<\omega}$ (resp. $\left.\alpha \rightarrow[\beta]_{\gamma,<\delta}^{<\omega}\right)$, we mean that for any $f:[\alpha]^{<\omega} \rightarrow P_{\gamma} \delta$ (resp. $f:[\alpha]^{<\omega} \rightarrow \gamma$ ) there exists $x \in[\alpha]^{\beta}$ with $\bigcup f^{\prime \prime}[x]^{<\omega} \neq \delta$ (resp. $\left|f^{\prime \prime}[x]^{<\omega}\right|<\delta$ ), where $\alpha, \beta, \gamma$ and $\delta$ are all cardinals.

Before introducing $\mu$-complete analogs of $C F_{\kappa \lambda}$ and $S C F_{\kappa \lambda}$, it is appropriate to recall the following characterizations of the original versions.

1.1. Proposition [1, 8]. $C F_{\kappa \lambda}$ (resp. $S C F_{\kappa \lambda}$ ) is generated by the sets of the form $\left\{x: f^{\prime \prime} x^{2} \subset P x\right\}$ (resp. $\left\{x: f^{\prime \prime} x \subset P x\right\}$ ), where $f: \lambda^{2} \rightarrow P_{\kappa} \lambda$ (resp. $\left.f: \lambda \rightarrow P_{\kappa} \lambda\right)$.

Definition. (1) $C F_{\kappa \lambda}^{\mu}$ (resp. $S C F_{\kappa \lambda}^{\mu}$ ) is the filter generated by the sets of the form $\left\{x: f^{\prime \prime} x^{2} \subset P x\right\}$ (resp. $\left.\left\{x: f^{\prime \prime} x \subset P x\right\}\right)$, where $f: \lambda^{2} \rightarrow P_{\mu} \lambda$ (resp. $\left.f: \lambda \rightarrow P_{\mu} \lambda\right)$.

(2) $X \subset P_{\kappa} \lambda$ is $\mu$-unbounded in $P_{\kappa} \lambda$ iff $X \cap P_{\mu} \lambda$ is unbounded in $P_{\mu} \lambda$.

The same argument as in $[1,2,4,8]$ works for the following (see [10] for a short proof of (2)).

1.2. Proposition. (1) $C F_{\kappa \lambda}^{\mu}$ (resp. $S C F_{\kappa \lambda}^{\mu}$ ) is generated by the closed (resp. strongly closed) and $\mu$-unbounded subsets of $P_{\kappa} \lambda$.

(2) $C F_{\kappa \lambda}^{\mu}=S C F_{\kappa \lambda}^{\mu}+\left\{x: \pi^{\prime \prime} x^{2} \subset x\right\}$.

(3) $C F_{\kappa \lambda}^{\mu}$ is the minimal normal $\mu$-complete filter on $P_{\kappa} \lambda$.

(4) $C F_{\kappa \lambda}^{\mu}=C F_{\kappa \lambda}^{\omega}+\{x: x \cap \mu \in \mu\}$, where $\omega_{1}<\mu$.

Proof. Let us give a combinatorial proof of (4), which is a prototype of later arguments.

Fix $f: \lambda^{2} \rightarrow P_{\mu} \lambda$. We define $h: \lambda^{2} \rightarrow P_{\omega} \lambda$ and show $\{x: x \cap \mu \in$ $\mu$ and $\left.h^{\prime \prime} x^{2} \subset P x\right\} \subset\left\{x: f^{\prime \prime} x^{2} \subset P x\right\}$.

First define $g: \lambda^{3} \rightarrow \lambda$ by $g(\alpha, \beta, 0)=|f(\alpha, \beta)|,\{g(\alpha, \beta, \gamma): 0<\gamma \leq$ $|f(\alpha, \beta)|\}=f(\alpha, \beta)$ and $g(\alpha, \beta, \gamma)=0$ for $\gamma>|f(\alpha, \beta)|$. We show $\left\{x: x \cap \mu \in \mu\right.$ and $\left.g^{\prime \prime} x^{3} \subset x\right\} \subset\left\{x: f^{\prime \prime} x^{2} \subset P x\right\}$.

Let $x \in P_{\kappa} \lambda$ be closed under $g$ and $x \cap \mu \in \mu$. Fix $\alpha, \beta \in x$. Then $0 \in x$, since $x \cap \mu \neq 0$ is an ordinal when $\alpha<\mu$ and since $0=g(\alpha, \alpha, \alpha)$ when $\alpha \geq \mu$. Hence $|f(\alpha, \beta)|+1 \subset x$, since $|f(\alpha, \beta)|=g(\alpha, \beta, 0) \in x \cap \mu$ is an ordinal. Thus $f(\alpha, \beta) \subset\{g(\alpha, \beta, \gamma): \gamma \leq|f(\alpha, \beta)|\} \subset x$.

Define $h: \lambda^{2} \rightarrow P_{\omega} \lambda$ by $h(\alpha, \beta)=\left\{\pi(\alpha, \beta), g\left(\alpha, \pi^{-1}(\beta)\right)\right\}$. Then $\{x:$ $\left.h^{\prime \prime} x^{2} \subset P x\right\} \subset\left\{x: g^{\prime \prime} x^{3} \subset x\right\}$ by the standard argument and hence we are done.

Several people $[2,4,6,9]$ have already characterized $C F_{\kappa \lambda}^{\omega_{1}}$ or $C F_{\omega_{1} \lambda}$ in their own ways. Let us summarize some of them.

1.3. Proposition. The following are equivalent for $X \subset P_{\kappa} \lambda$.

(1) $X \in C F_{\kappa \lambda}^{\omega}$.

(2) $X \in C F_{\kappa \lambda}^{\omega_{1}}$.

(3) $\left\{x: f^{\prime \prime} x^{<\omega} \subset x\right\} \subset X$ for some $f: \lambda^{<\omega} \rightarrow \lambda$.

(4) $\left\{x: f^{\prime \prime} x^{<\omega} \subset P x\right\} \subset X$ for some $f: \lambda^{<\omega} \rightarrow P_{\omega_{1}} \lambda$.

(5) $\left.\left\{x: x<\left(\lambda ; f_{i}\right)_{i<\omega}\right)\right\} \subset X$ for some structure $\left(\lambda ; f_{i}\right)_{i<\omega}$ with the universe 
$\lambda$ and finitary functions $\left\{f_{i}: i<\omega\right\}$ on $\lambda$, where " $<$ " denotes an elementary substructure.

Proof. We show only equivalence of (1) and (2). The other statements are equivalent to (2) by standard arguments.

Define $f: \lambda \rightarrow P_{\omega} \lambda$ by $f(\alpha)=\{0, \alpha+1\}$. Then $\left\{x: f^{\prime \prime} x \subset P x\right\} \subset\{x:$ $\omega \subset x\} \in C F_{\kappa \lambda}^{\omega}$. Hence $C F_{\kappa \lambda}^{\omega}$ is $\omega_{1}$-complete by the standard argument using normality of $C F_{\kappa \lambda}^{\omega}$. Thus $C F_{\kappa \lambda}^{\omega_{1}} \subset C F_{\kappa \lambda}^{\omega}$ by minimality of $C F_{\kappa \lambda}^{\omega_{1}}$.

\section{THE CASE $\mu=\omega_{1}$}

In this section, we try to find the simplest form of generators of $C F_{\kappa \lambda}^{\omega_{1}}$ and $S C F_{\kappa \lambda}^{\omega_{1}}$. It is easily seen by the methods in the last section that $C F_{\kappa \lambda}^{\omega_{1} \lambda}$ can be characterized by two binary functions on $\lambda$. A closer coding yields that one binary function suffices.

2.1. Theorem. $X \in C F_{\kappa \lambda}^{\omega_{1}}$ iff $\left\{x: f^{\prime \prime} x^{2} \subset x\right\} \subset X \subset P_{\kappa} \lambda$ for some $f: \lambda^{2} \rightarrow \lambda$. Proof. We show the only-if part.

Fix $f: \lambda^{2} \rightarrow P_{\omega} \lambda$. By Proposition 1.3, it suffices to define $p: \lambda^{2} \rightarrow \lambda$ and show $\left\{x: p^{\prime \prime} x^{2} \subset x\right\} \subset\left\{x: f^{\prime \prime} x^{2} \subset P x\right\}$.

First fix $\rho: \lambda^{2} \rightarrow \lambda$ such that $\rho \mid\left\{(\alpha, \beta) \in \lambda^{2}: \alpha \neq \beta\right\}$ is a bijection to $\lambda-\{0\}, \rho(\alpha, \alpha)=0$ for $\alpha<\lambda, \rho(\alpha, 0)$ is a limit ordinal for $0<\alpha<\lambda$, and $\rho(\alpha, \beta) \geq \omega$ for $\alpha<\lambda$ and $\beta<\omega$ with $\alpha \neq \beta$.

Define $g: \lambda \rightarrow P_{\omega} \lambda$ by $g(0)=f(0,0)$ and $g(\gamma)=f\left(\rho^{-1}(\gamma)\right) \cup f(\gamma, \gamma)$ for $\gamma>0$. We show $\left\{x: \rho^{\prime \prime} x^{2} \subset x\right.$ and $\left.g^{\prime \prime} x \subset P x\right\} \subset\left\{x: f^{\prime \prime} x^{2} \subset P x\right\}$.

Let $x \in P_{\kappa} \lambda$ be closed under $\rho$ and $g$. Fix $\alpha, \beta \in x$. Then $f(\alpha, \beta) \subset x$, since $f(\alpha, \beta) \subset g(\rho(\alpha, \beta))$ for $\alpha \neq \beta$, and since $f(\alpha, \alpha) \subset g(\alpha)$.

For $\alpha<\lambda$, we can fix $n_{\alpha}<\omega$ such that $n \neq \alpha$ and $\rho(\alpha, n) \notin g(\alpha)$ for any $n_{\alpha} \leq n<\omega$, since $\{n<\omega: \rho(\alpha, n) \in g(\alpha)\}$ is finite. Define $h: \lambda^{2} \rightarrow \lambda$ by $h(\alpha, 0)=\alpha+1,\left\{h(\alpha, n): n_{\alpha} \leq n<n_{\alpha}+|g(\alpha)|\right\}=g(\alpha)$ and $h(\alpha, \beta)=0$ for $0<\beta<n_{\alpha}$ and $n_{\alpha}+|g(\alpha)| \leq \beta$. We show $\left\{x: h^{\prime \prime} x^{2} \subset x\right\} \subset\left\{x: g^{\prime \prime} x \subset P x\right\}$.

Let $x \in P_{\kappa} \lambda$ be closed under $h$. Fix $\alpha \in x$. Then $\omega \subset x$, since $0=h(\alpha, \alpha)$ for $\alpha>0$ and $n+1=h(n, 0)$ for $n<\omega$. Thus $g(\alpha) \subset\{h(\alpha, n): n<\omega\} \subset$ $x$.

Define $k: \lambda \rightarrow \lambda$ by $k(0)=h(0,0)$ and $k(\gamma)=h\left(\rho^{-1}(\gamma)\right)$ for $\gamma>0$. We show $\left\{x: \rho^{\prime \prime} x^{2} \subset x\right.$ and $\left.k^{\prime \prime} x \subset x\right\} \subset\left\{x: h^{\prime \prime} x^{2} \subset x\right\}$.

Let $x \in P_{\kappa} \lambda$ be closed under $\rho$ and $k$. Fix $\alpha, \beta \in x$. Then $h(\alpha, \beta) \in x$, since $h(\alpha, \beta)=k(\rho(\alpha, \beta))$ for $\alpha \neq \beta$, since $h(\alpha, \alpha)=\rho(\alpha, \alpha)=0$ for $\alpha>0$ and since $h(0,0)=k(0)$.

Define $p: \lambda^{2} \rightarrow \lambda$ by $p(\alpha, \alpha)=k(\alpha)$ and $p(\alpha, \beta)=\rho(\alpha, \beta)$ for $\alpha \neq \beta$. We show $\left\{x: p^{\prime \prime} x^{2} \subset x\right\} \subset\left\{x: \rho^{\prime \prime} x^{2} \subset x\right.$ and $\left.k^{\prime \prime} x \subset x\right\}$.

Let $x \in P_{\kappa} \lambda$ be closed under $p$. Fix $\alpha \in x$. Then $k(\alpha)=p(\alpha, \alpha) \in x$ and $\rho(\alpha, \beta)=p(\alpha, \beta) \in x$ for $\beta \in x$ with $\alpha \neq \beta$. We show $\rho(\alpha, \alpha)=0 \in x$.

We can assume $\alpha>0$. Put $\delta=h(\beta, \gamma)$, where $\rho(\beta, \gamma)=\alpha$ and $\beta \neq \gamma$. Then $\delta=k(\alpha) \in x$ and $\delta \neq \alpha$, since $h(\beta, \gamma) \neq \rho(\beta, \gamma)$ by the definition of $h$ and $\rho$. Put $\eta=\rho(\delta, \alpha)$. Then $\eta>0$ and $\eta=p(\delta, \alpha) \in x$, since $\delta \neq \alpha$. Hence by the definition of $h, 0=h(\delta, \alpha)=k(\eta) \in x$ when $\alpha \geq \omega$. When $\alpha<\omega$, we have $\eta \geq \omega$ by the definition of $\rho$. Put $\theta=\rho(\alpha, \eta)$. Then $\theta>0$ and $\theta=p(\alpha, \eta) \in x$, since $\alpha \neq \eta$. Hence by the definition of $h$, $0=h(\alpha, \eta)=k(\theta) \in x$, since $\eta \geq \omega$. 
2.2. Corollary [7]. (1) $X \in C F_{\kappa \lambda}^{\mu}$ iff $\left\{x: x \cap \mu \in \mu\right.$ and $\left.f^{\prime \prime} x^{2} \subset x\right\} \subset X \subset P_{\kappa} \lambda$ for some $f: \lambda^{2} \rightarrow \lambda$.

(2) $X \in C F_{\omega_{1} \lambda}$ iff $\left\{x: f^{\prime \prime} x^{2} \subset x\right\} \subset X \subset P_{\omega_{1}} \lambda$ for some $f: \lambda^{2} \rightarrow \lambda$.

We need to introduce the successor function $\sigma: \lambda \rightarrow \lambda$ to get an analog of Theorem 2.1 for $S C F_{\kappa \lambda}^{\omega_{1}}$.

2.3. Theorem. $X \in S C F_{\kappa \lambda}^{\omega_{1}}$ iff $\left\{x: \sigma^{\prime \prime} x \subset x\right.$ and $\left.f^{\prime \prime} x \subset x\right\} \subset X \subset P_{\kappa} \lambda$ for some $f: \lambda \rightarrow \lambda$.

Proof. We show the only-if part.

Fix $f: \lambda \rightarrow P_{\omega_{1}} \lambda$. Define $g: \lambda \rightarrow \lambda$ by $g(\omega \cdot \alpha+n)=\omega \cdot \alpha$ for odd $n<\omega$ and $\{g(\omega \cdot \alpha+n): n<\omega$ is even $\}=\bigcup_{m<\omega} f(\omega \cdot \alpha+m)$. We show $\left\{x: \sigma^{\prime \prime} x \subset x\right.$ and $\left.g^{\prime \prime} x \subset x\right\} \subset\left\{x: f^{\prime \prime} x \subset P x\right\}$.

Let $x \in P_{\kappa} \lambda$ be closed under $\sigma$ and $g$. Fix $\omega \cdot \alpha+l \in x$ with $l<\omega$. Then $\omega \cdot \alpha \in x$, since $\omega \cdot \alpha=g(\omega \cdot \alpha+l)$ or $g(\sigma(\omega \cdot \alpha+l))$ according to whether $l$ is odd or even. Hence $\{\omega \cdot \alpha+n: n<\omega\} \subset x$, since $x$ is closed under $\sigma$. Thus $f(\omega \cdot \alpha+l) \subset \bigcup_{m<\omega} f(\omega \cdot \alpha+m) \subset\{g(\omega \cdot \alpha+n): n<\omega\} \subset x$.

2.4. Corollary. (1) $X \in S C F_{\omega_{1} \lambda}$ iff $\left\{x: \sigma^{\prime \prime} x \subset x\right.$ and $\left.f^{\prime \prime} x \subset x\right\} \subset X \subset P_{\omega_{1}} \lambda$ for some $f: \lambda \rightarrow \lambda$.

(2) $S C F_{\kappa \lambda}^{\omega_{1}}=S C F_{\kappa \lambda}^{\omega}$.

A simple observation yields that Theorem 2.3 is optimal with respect to the number of characterizing functions.

2.5. Proposition. $\left\{x: f^{\prime \prime} x \subset x\right\} \not \subset\{x: \omega \subset x\}$ for any $f: \lambda \rightarrow \lambda$.

Proof. Let $f: \lambda \rightarrow \lambda$ be a counterexample. Set $x=\left\{f^{m}(0): 0<m<\omega\right\}$, where $f^{m}: \lambda \rightarrow \lambda$ is the $m$-fold iteration of $f$. Then $\omega \subset x$, since $x$ is closed under $f$ and by the choice of $f$. Pick $0<n<\omega$ with $f^{n}(0)=0$. Then $x=\left\{f^{m}(0): 0<m \leq n\right\}$ is finite, contradicting to $\omega \subset x$.

\section{THE CASE $\mu>\omega_{1}$}

We have already seen in the last section that $C F_{\kappa \lambda}^{\omega_{1}}=C F_{\kappa \lambda}^{\omega}$ and $S C F_{\kappa \lambda}^{\omega_{1}}=$ $S C F_{\kappa \lambda}^{\omega}$. This never happens for the pair $(\nu, \mu)$ in place of $\left(\omega_{1}, \omega\right)$. Instead we have four kinds of simply definable subsets of $P_{\kappa} \lambda$ which witness the difference between $C F_{\kappa \lambda}^{\nu}\left(\right.$ resp. $\left.S C F_{\kappa \lambda}^{\nu}\right)$ and $C F_{\kappa \lambda}^{\mu}$ (resp. $\left.S C F_{\kappa \lambda}^{\mu}\right)$ as a candidate for a generator of the former over the latter.

The same argument as in Theorem 2.3 settles the first case where $\nu=\xi^{+}$ and the candidate is $\left\{x: \sigma_{\xi}^{\prime \prime} x \subset P x\right\}$.

3.1. Theorem. $X \in S C F_{\kappa \lambda}^{\xi^{+}}$iff $\left\{x: \sigma_{\xi}^{\prime \prime} x \subset P x\right.$ and $\left.f^{\prime \prime} x \subset x\right\} \subset X \subset P_{\kappa} \lambda$ for some $f: \lambda \rightarrow \lambda$.

3.2. Corollary. (1) $S C F_{\kappa \lambda}^{\xi^{+}}=S C F_{\kappa \lambda}^{\omega}+\left\{x: \sigma_{\xi}^{\prime \prime} x \subset P x\right\}$.

(2) $C F_{\kappa \lambda}^{\xi^{+}}=C F_{\kappa \lambda}^{\omega}+\left\{x: \sigma_{\xi}^{\prime \prime} x \subset P x\right\}$.

We have already seen in Proposition 1.2(4) the second case for $C F_{\kappa \lambda}^{\mu}$ where the candidate is $\{x: x \cap \nu \in \nu\}$. An analog of Proposition 1.2(4) fails for $S C F_{\kappa \lambda}^{\mu}$ as in Corollary 3.4(4). We have in fact the following stronger result. 
3.3. Theorem. $S C F_{\kappa \lambda}^{\mu^{+}} \not \subset S C F_{\kappa \lambda}^{\mu}+\{x: x \cap \kappa \in \kappa\}$.

Proof. We show $\left\{x: \sigma_{\mu}^{\prime \prime} x \subset P x\right\} \notin S C F_{\kappa \lambda}^{\mu}+\{x: x \cap \kappa \in \kappa\}$.

Otherwise pick $f: \lambda \rightarrow P_{\mu} \lambda$ with $\left\{x: x \cap \kappa \in \kappa\right.$ and $\left.f^{\prime \prime} x \subset P x\right\} \subset\{x:$ $\left.\sigma_{\mu}^{\prime \prime} x \subset P x\right\}$.

Let $x \in[\lambda]^{\kappa}$ be the closure of $\kappa$ under $f$. Then we can pick $\beta=\mu \cdot \alpha<\lambda$ with $\sigma_{\mu}(\beta) \cap x=0$, since $\lambda$ is the disjoint union of $\left\{\sigma_{\mu}(\mu \cdot \alpha): \alpha<\lambda\right\}$. Let $y \in P_{\mu} \lambda$ be the closure of $\{\beta\}$ under $f$. Set $z=\bigcup_{n<\omega} z_{n}$, where $z_{n} \in P_{\kappa} \lambda$ is defined inductively by $z_{0}=\{\beta\}$ and $z_{n+1}=\sup \left(z_{n} \cap \kappa\right) \cup \cup f^{\prime \prime} z_{n}$. Then $\sigma_{\mu}(\beta) \subset z$, since $z \cap \kappa \in \kappa$ and $z$ is closed under $f$ and by the choice of $f$. On the other hand $z \subset x \cup y$, since $z_{n} \subset x \cup y$ by induction on $n<\omega$. Hence $\sigma_{\mu}(\beta) \subset y$, since $\sigma_{\mu}(\beta) \cap x=0$, contradicting to $y \in P_{\mu} \lambda$.

3.4. Corollary. Let $\mu^{+}<\nu$ in (1) and $\mu<\xi$ in (2) and (3).

(1) $S C F_{\kappa \lambda}^{\mu^{+}}+\{x: x \cap \nu \in \nu\} \supsetneq S C F_{\kappa \lambda}^{\mu}+\{x: x \cap \nu \in \nu\}$.

(2) $S C F_{\kappa \lambda}^{\mu^{+}}+\{x: \xi \subset x\} \supsetneq S C F_{\kappa \lambda}^{\mu}+\{x: \xi \subset x\}$.

(3) $S C F_{\kappa \lambda}^{\mu^{+}}+\{x:|x| \geq \xi\} \supsetneq S C F_{\kappa \lambda}^{\mu}+\{x:|x| \geq \xi\}$.

(4) $S C F_{\kappa \lambda}^{\nu} \supsetneq S C F_{\kappa \lambda}^{\mu}+\{x: x \cap \nu \in \nu\}$.

(5) $S C F_{\kappa \lambda}^{\xi^{+}} \supsetneq S C F_{\kappa \lambda}^{\mu}+\{x: \xi \subset x\}$.

(6) $S C F_{\kappa \lambda}^{\xi^{+}} \supsetneq S C F_{\kappa \lambda}^{\mu}+\{x:|x| \geq \xi\}$.

The same argument as in Proposition 1.3 settles the third case for $C F_{\kappa \lambda}^{\mu}$ where $\nu=\xi^{+}$and the candidate is $\{x: \xi \subset x\}$.

3.5. Proposition. $C F_{\kappa \lambda}^{\xi^{+}}=C F_{\kappa \lambda}^{\omega}+\{x: \xi \subset x\}$.

An analog of Proposition 3.5 fails for $S C F_{\kappa \lambda}^{\mu}$ as in Corollary 3.4(5). We have in fact the following stronger result.

3.6. Theorem. Let $\mu \leq \xi$. Then $S C F_{\kappa \lambda}^{\mu}+\left\{x: x \cap \xi^{+} \in \xi^{+}\right\} \supsetneq S C F_{\kappa \lambda}^{\mu}+\{x: \xi \subset$ $x\}$.

Proof. We show $\left\{x: x \cap \xi^{+} \in \xi^{+}\right\} \notin S C F_{\kappa \lambda}^{\mu}+\{x: \xi \subset x\}$. $\left.\xi^{+}\right\}$.

Otherwise pick $f: \lambda \rightarrow P_{\mu} \lambda$ with $\left\{x: \xi \subset x\right.$ and $\left.f^{\prime \prime} x \subset P x\right\} \subset\left\{x: x \cap \xi^{+} \in\right.$

Let $x \in[\lambda]^{\xi}$ be the closure of $\xi$ under $f$. Set $\beta=x \cap \xi^{+} \in \xi^{+}$. Let $y \in P_{\mu} \lambda$ be the closure of $\{\beta+\xi\}$ under $f$. Then $\beta+\xi \in(x \cup y) \cap \xi^{+}$is an ordinal, since $\xi \subset x \cup y$ is closed under $f$ and by the choice of $f$. Hence $\beta+\xi \subset x \cup y$. Thus $\{\beta+\gamma: \gamma<\xi\} \subset y$, since $x \cap(\beta+\xi) \subset \beta$, contradicting to $y \in P_{\mu} \lambda$.

We need to generalize the notion of square bracket partition relations (see [3]) to consider the forth case for $C F_{\kappa \lambda}^{\mu}$ where $\nu=\xi^{+}$and the candidate is $\{x:|x| \geq \xi\}$.

3.7. Theorem. Let $\mu \leq \zeta \leq \xi$. Then $C F_{\kappa \lambda}^{\zeta^{+}} \subset C F_{\kappa \lambda}^{\mu}+\{x:|x| \geq \xi\}$ iff $\lambda \nrightarrow[\xi]_{P_{\mu} \zeta}^{<\omega}$.

Proof. First observe that $C F_{\kappa \lambda}^{\zeta^{+}} \subset C F_{\kappa \lambda}^{\mu}+\{x:|x| \geq \xi\}$ iff $\{x: \zeta \subset x\} \in C F_{\kappa \lambda}^{\mu}+$ $\{x:|x| \geq \xi\}$ iff there exists $f: \lambda^{2} \rightarrow P_{\mu} \lambda$ with $\left\{x:|x| \geq \nu\right.$ and $\left.f^{\prime \prime} x^{2} \subset P x\right\} \subset$ $\{x: \zeta \subset x\}$ iff there exists $f: \lambda^{2} \rightarrow P_{\mu} \lambda$ with $\zeta \subset x_{y}$ for any $y \in[\lambda]^{\xi}$, where 
$x_{z} \in P_{\kappa} \lambda$ is defined to be the closure of $z \in P_{\kappa} \lambda$ under $f$ iff $(*)$ there exists $f: \lambda^{2} \rightarrow P_{\mu} \lambda$ with $\bigcup g^{\prime \prime}[y]^{<\omega}=\zeta$ for any $y \in[\lambda]^{\xi}$, where $g:[\lambda]^{<\omega} \rightarrow P_{\mu} \zeta$ is defined by $g(s)=x_{s} \cap \zeta$.

The last equivalence follows from the fact that $x_{z}=\bigcup_{s \in[z]^{<\omega}} x_{s}$ for any $z \in P_{\kappa} \lambda$, which we show.

Define $x_{z, n} \in P_{\kappa} \lambda$ inductively by $x_{z, 0}=z$ and $x_{z, n+1}=x_{z, n} \cup \bigcup f^{\prime \prime} x_{z, n}^{2}$. Then $x_{z}=\bigcup_{n<\omega} x_{z, n}$ and $x_{z, n} \subset \bigcup_{s \in[z]^{<\omega}} x_{s}$ by induction on $n<\omega$.

Now we show that $(*)$ holds iff $\lambda \nrightarrow[\xi]_{P_{\mu} \zeta}^{<\omega}$.

If part. Let $h:[\lambda]^{<\omega} \rightarrow P_{\mu} \zeta$ witness $\lambda \nrightarrow[\xi]_{P_{\mu} \zeta}^{<\omega}$. Define $f: \lambda^{2} \rightarrow P_{\mu} \lambda$ by $f(\alpha, \beta)=\{\pi(\alpha, \beta)\} \cup \cup h^{\prime \prime}\left[y_{\alpha}\right]^{<\omega}$, where $y_{\alpha} \in P_{\omega_{1}} \lambda$ is the closure of $\{\alpha\}$ under $\pi^{-1}$. We show that $h(s) \subset x_{s}$ for any $s \in[\lambda]^{<\omega}-\{0\}$, which immediately implies that $f$ witnesses $(*)$.

Fix $s=\left\{\alpha_{i}: i<n\right\} \in[\lambda]^{<\omega}$. Define $\beta_{i}<\lambda$ inductively for $i<n$ by $\beta_{0}=\alpha_{0}$ and $\beta_{i+1}=\pi\left(\beta_{i}, \alpha_{i+1}\right)$. Then $\beta_{i} \in x_{s}$ by induction on $i<n$, since $x_{s}$ is closed under $\pi$. Thus $h(s) \subset f\left(\beta_{n}, \beta_{n}\right) \subset x_{s}$, since $s \in\left[y_{\beta_{n}}\right]^{<\omega}$.

Only-if part. $g:[\lambda]^{<\omega} \rightarrow P_{\mu} \zeta$ defined as in $(*)$ clearly witnesses $\lambda \nrightarrow$ $[\xi]_{P_{\mu} \zeta}^{<\omega}$.

It is easily seen that $\lambda \rightarrow[\xi]_{P_{\mu} \mu}^{<\omega}$ iff $\lambda \rightarrow[\xi]_{\mu,<\mu}^{<\omega}$ a general form of Chang's conjecture (see [3]).

3.8. Corollary. Let $\mu \leq \xi$. Then

(1) $C F_{\kappa \lambda}^{\mu^{+}}+\{x:|x| \geq \xi\}=C F_{\kappa \lambda}^{\mu}+\{x:|x| \geq \xi\}$ iff $\lambda \nrightarrow[\xi]_{\mu,<\mu}^{<\omega}$.

(2) $C F_{\kappa \lambda}^{\xi^{+}}=C F_{\kappa \lambda}^{\mu}+\{x:|x| \geq \xi\}$ iff $\lambda \nrightarrow[\xi]_{P_{\mu} \xi}^{<\omega}$.

An analog of Corollary 3.8(2) fails for $S C F_{\kappa \lambda}^{\mu}$ regardless of partition relations as in Corollary 3.4(6). We have in fact the following stronger result.

3.9. Theorem. Let $\mu \leq \xi$. Then $S C F_{\kappa \lambda}^{\mu}+\{x: \xi \subset x\} \supsetneq S C F_{\kappa \lambda}^{\mu}+\{x:|x| \geq \xi\}$. Proof. We show $\{x: \xi \subset x\} \notin S C F_{\kappa \lambda}^{\mu}+\{x:|x| \geq \xi\}$.

Otherwise pick $f: \lambda \rightarrow P_{\mu} \lambda$ with $\left\{x:|x| \geq \xi\right.$ and $\left.f^{\prime \prime} x \subset P x\right\} \subset\{x: \xi \subset x\}$.

Let $x_{\alpha} \in P_{\mu} \lambda$ be the closure of $\{\alpha\}$ under $f$ for $\alpha<\lambda$. Define $g: \lambda \rightarrow \mu$ by $g(\alpha)=\sup \left(x_{\alpha} \cap \mu\right)+1$. Pick $y \in[\lambda]^{\xi}$ and $\gamma<\mu$ with $g^{\prime \prime} y=\{\gamma\}$. Then $z=\bigcup_{\alpha \in y} x_{\alpha} \in[\lambda]^{\xi}$ is closed under $f$ and $z \cap \mu \subset \bigcup g^{\prime \prime} y \subset \gamma$, contradicting to the choice of $f$.

\section{REFERENCES}

1. D. M. Carr, The minimal normal filter on $P_{\kappa} \lambda$, Proc. Amer. Math. Soc. 86 (1982), 316-320.

2. H.-D. Donder, P. Koepke, and J.-P. Levinski, Some stationary subsets of $P_{\kappa} \lambda$, Proc. Amer. Math. Soc. 102 (1988), 1000-1004.

3. P. Erdös, A. Hajnal, A. Máté, and R. Rado, Combinatorial set theory: partition relations for cardinals, Stud. Logic Found. Math., vol. 106, North-Holland, Amsterdam, 1984.

4. M. Foreman, M. Magidor, and S. Shelah, Martin's Maximum, saturated ideals and nonregular ultrafilters, part I, Ann. of Math. (2) 127 (1988), 1-47.

5. T. J. Jech, Some combinatorial problems concerning uncountable cardinals, Ann. Maih. Logic 5 (1973), 165-198.

6. D. W. Kueker, Countable approximations and Löwenheim-Skolem theorems, Ann. Math. Logic 11 (1977), 57-103. 
7. P. Matet, Concerning stationary subsets of $[\lambda]^{<\kappa}$, Set Theory and its Applications (J. Steprāns and S. Watson, eds.), Lecture Notes in Math., vol. 1401, Springer, Berlin, Heidelberg, and New York, 1989, pp. 119-127.

8. T. K. Menas, On strong compactness and supercompactness, Ann. Math. Logic 7 (1974), 327-359.

9. S. Shelah, Proper forcing, Lecture Notes in Math., vol. 940, Springer, Berlin, Heidelberg, and New York, 1982.

10. M. Shioya, M., Weakly normal closures of filters on $P_{\kappa} \lambda$, J. Symbolic Logic 58 (1993), 55-63.

INSTItUTe of Mathematics, UNIVERsity of TsukUba, Tsukuba, Ibaraki, 305 Japan

E-mail address: shioyacsakura.cc.tsukuba.ac.jp 